\title{
Lactente acometida pela síndrome de Guillain-Barré em hospital regional filantrópico do sudoeste de Minas Gerais
}

Infant affected by Guillain-Barré syndrome in a local philanthropic hospital of Minas Gerais southwest

Niña acometida por el Síndrome de Guillain-Barré en hospital regional filántropo del sudoeste de Minas Gerais

Júlio Henrique Muzetti* ${ }^{1 *}$, Paulo Prado Vasconcelos ${ }^{1}$, Gustavo Henrique Reis Mariano², Anne Laryana Veloso Moura Alvarenga ${ }^{1}$, Thais Moreno Rodrigues ${ }^{1}$.

\section{RESUMO}

Objetivo: Relatar o acometimento de uma lactente pela Síndrome de Guillain-Barré, a conduta adotada e a sua resposta ao tratamento em um Hospital Regional Filantrópico do Sudoeste do Estado de Minas Gerais. Relato: Realizou-se 0 atendimento da paciente no hospital referido pela equipe pediátrica em multidisciplinaridade com neurologistas, fisioterapeutas e fonoaudiólogos. $\mathrm{O}$ atendimento proporcionou um tratamento de qualidade para a paciente, resultando em uma recuperação adequada e satisfatória, aparentemente sem prejuízos no pós-hospitalar. Conclusão: As informações ainda deficitárias sobre a Síndrome de Guillain-Barré, do ponto de vista etiológico, diagnóstico, de tratamento e prevenção, fazem com que o tratamento de pacientes acometidos por tal patologia seja, muitas vezes, deficitário. Por isso, este estudo foi importante para que outras pessoas possam receber um diagnóstico precoce, além de um tratamento multidisciplinar e com bons resultados.

Palavras-chave: Síndrome de Guillain-Barré, Polineuropatia, Fraqueza muscular.

\begin{abstract}
Objective: Reporting a case of an infant affected by Guillain-Barré syndrome, the conduct adopted and her treatment response in a philanthropic local hospital of Minas Gerais southwest. Report: The child received the hospital attention by the pediatric team with other professionals like neurologists, physiotherapists and phonoaudiologists. The attention provided a quality treatment to the patient, resulting in an appropriate and a satisfactory recovery, apparently without injuries after the discharge from the hospital. Conclusion: The information about the Guillain-Barré syndrome is still having a deficit, from the etiologic, diagnostic, treatment and prevention viewpoints, making the patients treatment affected by Guillain-Barré syndrome to be not good in many times. Therefore, the present study was important to other people affected by this pathology so that they can receive a precocious diagnosis, as well a multidisciplinary treatment with good results.
\end{abstract}

Keywords: Guillain-Barré syndrome, Polyneuropathy, Muscular weakness.

\section{RESUMEN}

Objetivo: Relatar el acometimiento de una niña por el Síndrome de Guillain-Barré, la conducta adoptada y su respuesta a el tratamiento en un Hospital Regional Filántropo del sudoeste de Minas Gerais. Relato: Fue realizado el atendimiento del paciente en el hospital dicho por el equipo pediátrico junto con otros profesionales como neurólogos, fisioterapeutas y logopedas. El atendimiento puede proporcionar un tratamiento de cualidad para la niña, resultando en una recuperación adecuada y satisfactoria, aparentemente sin pérdidas considerables después de la salida del hospital. Conclusión: Las pocas informaciones sobre el Síndrome de Guillain-Barré, del punto de vista etiológico, diagnóstico, de tratamiento y prevención, hacen con que el tratamiento de pacientes afligidos por esta patología ser poco en muchas veces. Por eso, este estudio fue importante para que otras personas puedan recibir un diagnóstico precoz, además de un tratamiento con muchos profesionales y con buenos resultados.

Palabras clave: Síndrome de Guillain-Barré, Poli neuropatía, Debilidad muscular.

\footnotetext{
${ }^{1}$ Santa Casa de Misericórdia de Passos. Passos - MG. *E-mail: juliomuzetti@gmail.com

2 Universidade do Vale do Sapucaí (UNIVÁS). Pouso Alegre - MG.
} 


\section{INTRODUÇÃO}

A Síndrome de Guillain-Barré é uma patologia que foi descrita pela primeira vez por Landry no século XIX, e a partir do relato de Guillain-Barré e Strohi, em 1916, é que foi clinicamente conceituada (BENETI GM, SILVA DLD, 2006). Sua ocorrência é devido a uma desordem do Sistema Imunológico, quando este gera uma inflamação, no Sistema Nervoso Periférico, depois de atacá-lo. Isto ocorre, geralmente, aproximadamente 10 dias após um quadro infeccioso viral inespecífico, o qual pode ter cursado apenas com sintomas gastrointestinais ou do trato respiratório. Porém, existem relatos de surgimento da Síndrome até mesmo após a administração de vacinas contra raiva e influenza, por exemplo. Assim, o sistema imunológico realiza um ataque à bainha de mielina desses nervos, com sua consequente desmielinização aguda (SEJVAR JJ, et al., 2011; SANTANA JCB, et al., 1996).

Além disso, também segundo SEJVAR JJ, et al. (2011), ela não é claramente definida, mas acredita-se que a semelhança estrutural entre as moléculas presentes na bainha de mielina dos neurônios à estrutura desses vírus leva à produção, por meio do sistema imunológico, de anticorpos que irão agir no sistema nervoso periférico e causar tal desmielinização. Dessa maneira, os impulsos nervosos levados por esses axônios e o funcionamento desses neurônios são prejudicados substancialmente. Isto gera um quadro de polineuropatia com perda progressiva da força muscular em membros inferiores e superiores, de maneira ascendente e que tende à simetria de ambos os lados e arreflexia e parestesia dos membros. (CABRAL EKF, et al., 2012).

De acordo com MALTA JMAS, et al. (2017), estima-se que a síndrome de Guillain-Barré tenha uma incidência de aproximadamente 0,81 a 1,89 casos para cada 100.000 habitantes na população geral, sem correlação com sazonalidade e com um aparente aumento gradual de tal incidência de acordo com o avançar da idade. A sua história natural tem uma apresentação clínica grave, embora a sua evolução seja, na maioria dos casos, autolimitada, com bom prognóstico, baixo índice de mortalidade nos dias atuais e esta, quando ocorre, se dá devido a eventos infecciosos e trombóticos, e não mais devido a insuficiência respiratória (SANTANA JCB, et al., 1996; CABRAL EKF, et al., 2012).

Embora ela possa ocorrer em qualquer idade, não há, no entanto, grandes incidências dessa patologia em crianças abaixo dos doze meses ou próximas a um ano de idade ( $20 \%$ dos casos). e sim, entre os 20 e 40 anos, algo que faz com que a abordagem desses pacientes e seus tratamentos sejam realizados com certa insegurança por parte de profissionais menos habituados ou de centros de tratamento não especializados (BENETI GM, SILVA DLD, 2006; MALTA JMAS, et al., 2017).

Devido a isso, é de grande valia este relato, no qual os autores descrevem o caso de uma lactente acometida pela síndrome, ressaltando o seu diagnóstico, o seu tratamento, a sua evolução clínica e o seu prognóstico em um hospital de referência do interior do sudoeste de Minas Gerais.

\section{RELATO DE CASO}

O presente estudo relata o caso de uma paciente do sexo feminino de um ano e dez meses de idade que deu entrada em hospital de cidade de origem com quadro de fraqueza em membros inferiores com uma semana de evolução, mas sem referir febre ou outros sintomas que remetem a infecções. Na atual localidade, obteve a hipótese diagnóstica de Síndrome de Guillain-Barré e recebeu, portanto, uma dose de imonuglobulina, além de ter sido encaminhada ao hospital de referência para seguimento com departamento de neurologia.

Ao ser admitida pela equipe pediátrica no serviço de referência (Santa Casa de Misericórdia de Passos), a lactente apresentava quadro repentino de "perda das forças" em membros inferiores (mmll) com parestesia e arreflexia. Mostrava-se com progressão ascendente do quadro ao manifestar parestesia em membro superior esquerdo (mSE). Mãe referia, ainda, episódio de foliculite dois dias antes do início do quadro, mas negava vômitos, diarreia, febre ou outros sinais flogísticos. Ao exame físico a paciente estava em bom estado geral, anictérica, acianótica, afebril, normocorada, hidratada e cooperativa ao exame. Seu aparelho 
respiratório possuía murmúrio vesicular fisiológico presente bilateralmente e com ausência de ruídos adventícios. Seu aparelho cardiovascular expressava ritmo cardíaco regular, em dois tempos, sem a ausculta de sopros ou outros sons patológicos. Seu abdome estava normotenso, sem fácies de dor às palpações superficial e profunda, ruído hidroaéreo presente e dentro da normalidade, ausência de massas palpáveis ou visceromegalias. O mSE estava com força muscular reduzida e os $\mathrm{mmll}$ com pulsos periféricos palpáveis, mas hiporreflexivos e dolorosos à palpação.

Em seguida, a paciente foi avaliada pelo departamento de neurologia, recebendo o seguinte parecer: lactente interage com examinador, demonstra tetraparesia flácida associada à síndrome da cabeça caída, com arreflexia global e flacidez dos membros. Tal setor solicitou avaliação de líquor e recebeu como resultado a presença de três células e a dosagem de 61,9 de proteínas totais. Assim, após ter firmado o diagnóstico inicial de Síndrome de Guillain-Barré (sobretudo de acordo com os critérios de Asbury e Cornblath), tal equipe tomou como conduta a administração de $2,2 \mathrm{~g}$ de imunoglobulina humana $5 \mathrm{~g} / 100 \mathrm{~mL}$ por via endovenosa (EV) a cada $12 \mathrm{~h}$ e requisitou avaliação e acompanhamento do departamento de fisioterapia e fonoaudiologia do mesmo serviço de referência.

À avaliação da fisioterapia a paciente em questão mostrava-se com respiração espontânea em ar ambiente, leve taquipnéia e ausência de sinais de desconforto respiratório. Portanto, foram feitos exercícios passivos de mmll com descarga de peso, exercícios ativos de membros superiores (mmSS) e exercícios para controle de tronco e cabeça.

O departamento de fonoaudiologia, por sua vez, recebeu a lactente com ausência de ruídos anormais em ausculta cervical e presença de sucção e deglutição dentro dos padrões de normalidade. Além de ausência de tosse ou engasgos e deglutição funcional II segundo o Protocolo Fonoaudiólogo de Avaliação do risco para Disfagia (PARD), nível 5 da Escala Funcional de Ingestão por Via Oral (FOIS).

A paciente continuou sendo acompanhada diariamente pela equipe multiprofissional e com ótima evolução clínica. Alcançou, após seis dias de internação e com a administração de imunoglobulina, melhora significativa da força muscular e da dor, da sustentação da cabeça e dos movimentos ativos de mmSS e mmll. Por conseguinte, recebeu alta hospitalar após sete dias de internação e, quanto às orientações, foi recomendado a sua mãe retorno em trinta dias para a reavaliação e retorno imediato na vigência de intercorrências. Decorridos os trinta dias após a alta hospitalar, a paciente retornou, nesta ocasião, apresentava-se bem e sem sinais de perda de força muscular.

\section{DISCUSSÃO}

A fraqueza muscular aguda na criança é uma emergência pediátrica. Durante a abordagem diagnóstica, é de suma importância uma anamnese completa que inclua informações como: início da fraqueza, história de estados febris associados, ingestão de substâncias exógenas, imunizações e história familiar. O exame neurológico também deve ser acurado. Várias doenças possuem características semelhantes nas quais detalhes podem fazer a diferença para o diagnostico e para o prognostico do quadro. A investigação inicial na orientação do diagnóstico é de grande importância para melhor prognóstico da criança afetada, uma vez que as principais patologias cursam com características próprias como: miosite com aumento de creatina quinase (CK), paralisia periódica com hipopotassemia, miastenia gravis ou botulismo com ptose, oftalmoparesia e sinais bulbares e a Síndrome de Guillain-Barré, causa mais frequente de fraqueza muscular aguda cursando com fraqueza distal e hiporreflexia (TORRICELLI RPJE, 2017).

Asbury AK (2000) define a SGB como uma polirradiculoneuropatia autoimune aguda com a presença de paralisia flácida e arreflexia, alterações variáveis de sensibilidade e aumento de proteínas no líquor com ausência de pleocitose.

Esta patologia acomete tanto o sexo masculino quanto o feminino (com predomínio do masculino) e tem como faixa etária de maior incidência os adultos jovens entre os 20 a 30 anos. No entanto, $20 \%$ de todos os casos ocorrem em crianças com idade inferior a 10 anos (ASBURY AK, CORNBLATH DR, 1990; CECATTO S, et al., 2003). 
Pode se desenvolver de forma aguda ou subaguda, com evolução ao longo de dias ou semanas, apresentando-se de forma simétrica na grande maioria dos casos, mas com risco de evoluir de forma totalmente atípica. A SGB envolve principalmente os nervos motores sendo caracterizada por comprometimento periférico ascendente, com início geralmente nas extremidades inferiores e envolvendo, progressivamente, o tronco, os membros superiores e podendo acometer, finalmente, os músculos bulbares levando a insuficiência respiratória. Perda do controle esfincteriano, comprometimento de pares cranianos e diminuição dos reflexos tendinosos profundos podem estar associados. Os nervos sensitivos e autônomos também podem estar envolvidos apresentando instabilidade da pressão arterial e da frequência cardíaca, hipotensão postural, bradicardia e assistolia. Sinais relacionados a ventilação, deglutição e movimentos oculares demonstram uma maior gravidade do caso e exigem maiores cuidado e intervenção. As crianças geralmente ficam irritadas, com recusa ou impossibilidade de deambulação e evoluem com tetraplegia flácida e até tetraparesia, como a paciente em questão (DOORN P, et al., 2008; GOLDMAN L, SCHAFER Al, 2014; LONGO DL, et al., 2013).

As manifestações clínicas, em geral, são antecedidas por infecção viral inespecífica, preferencialmente respiratória ou intestinal, mas há relatos da síndrome após a administração de vacinas contra raiva, influenza, poliomielite (oral) e meningite conjugada. Com isso, acredita-se que a sua fisiopatologia seja sustentada por uma reação cruzada que ocorre com a formação de autoanticorpos, como os anti-glicofosfolipídeos 1 (presentes em $20 \%$ a $50 \%$ dos casos), que se ligam a epítopos nos nervos periféricos semelhantes àqueles encontrados na superfície de vírus e bactérias, como a Campylobacter jejuni, que podem ter causado tais processos inflamatórios prévios (NOVIELLO TB, et al., 2008; SEJVAR JJ, et al., 2011).

Com relação a prejuízos na fala e deglutição, embora a paciente não os tenha apresentado de maneira severa, eles estavam presentes nos níveis II (deglutição funcional) e 5 (via oral com múltiplas consistências, porém com necessidade de preparo especial ou compensações), de acordo com os critérios de PARD e FOIS, respectivamente.

Os aspectos clínicos utilizados para orientar e definir o diagnóstico da Síndrome de Guillain-Barré da paciente, bem como sua gravidade foram baseados nos propostos de Asbury e Cornblath de 1990, os quais são (1) aspectos necessários para o diagnóstico: perda progressiva da força em braços e pernas e arreflexia., (2) aspectos que sustentam fortemente o diagnóstico, como progressão dos sintomas em até quatro semanas, simetria relativa dos sintomas, ausência de febre no início e proteinorraquia elevada com celularidade liquórica abaixo de $10 \mathrm{cel} / \mathrm{mm}^{3}$, e (3) os que são de aspecto duvidoso são líquor com mais de $50 \mathrm{cel} / \mathrm{mm}^{3}$ e nível do sensório preservado. Já aqueles que excluem o diagnóstico são confirmação de botulismo ou miastenia, poliomielite ou neuropatia tóxica e metabolismo da porfirina anormal (ASBURY AK, CORNBLATH DR, 1990).

Portanto, pode-se perceber que há a confirmação do diagnóstico, uma vez que a paciente apresentou os dois itens necessários para o diagnóstico, quatro de um total de nove itens que sustentam fortemente 0 diagnóstico e nenhum dos três que o excluem. Além disso, o sucesso com apenas seis dias de tratamento com Imunoglobulina endovenosa também sustenta o diagnóstico.

Além de o diagnostico ser dado principalmente pelo quadro clínico, às vezes há a necessidade do auxílio de exames de imagem para a exclusão de outras patologias, como a síndrome medular. O exame de líquor apresenta uma alteração característica: dissociação albuminocitólogica (aumento da proteína sem aumento do número de células com a presença de apenas $3 c e l s / \mathrm{mm}^{3}$ e $61,9 \mathrm{mg} / \mathrm{dL}$ de proteínas), como citado anteriormente. Tal achado só estará presente após uma semana do início dos sintomas. A eletroneuromiografia (ENM) mostra uma polineuropatia com padrão desmielinizante na maioria dos casos (DOORN P, et al., 2008; GOLDMAN L, SCHAFER Al, 2014; LONGO DL, et al., 2013).

Assim, para o tratamento o paciente deve estar internado, preferencialmente em unidade de terapia intensiva (UTI), devendo-se realizar profilaxia contra tromboembolismo e infecções e manter cuidados gerais com a pele, além de suporte nutricional e fisioterapia motora para evitar contraturas. As causas mais comuns de óbito eram as complicações pulmonares secundárias a ventilação mecânica, no entanto, hoje em dia estas se são devido a infecções e eventos trombóticos, tal como afirma Cabral EKF, et al. (2012). O tratamento 
específico deve ser iniciado com imunoglobulina IV ou plasmaférese, pois ambas têm efeito rápido em doenças autoimunes. O objetivo do tratamento é reduzir o tempo de fraqueza muscular e impedir complicações graves, sendo que tanto a imunoglobulina IV quanto a plasmaférese possuem eficácia semelhante. O uso de corticoides não apresenta benefícios (DOORN P, et al., 2008; LONGO DL, et al., 2013).

\section{CONCLUSÃO}

As informações acerca da SGB ainda são deficitárias do ponto de vista etiológico, diagnóstico, de tratamento e prevenção; no entanto, por ser uma patologia importante, de incidência crescente, tal trabalho abrangeu de forma rápida e simples informações a respeito desta doença, para que ela possa ser cada vez mais conhecida, entendida e estudada. O caso relatado tem relevância devido à idade da paciente acometida, além de mostrar a importância do diagnóstico e tratamento precoces para uma boa evolução e prognóstico. Uma equipe multidisciplinar presente, ativa e que trabalha em conjunto interfere diretamente numa evolução favorável com menor tempo de internação e complicações associadas.

\section{REFERÊNCIAS}

1. ASBURY AK. New concepts of Guillain-Barré syndrome. Journal of Child Neurology, 2000; 15(3): 183-191.

2. ASBURY AK, CORNBLATH DR. Assessment of current diagnostic criteria for Guillain Barré syndrome. Annals of Neurology, 1990; 27: S21-S24.

3. BENETI GM, SILVA DLD. Síndrome de Guillain-Barré. Semina: Ciências Biológicas e Saúde, 2006; 27(1): 5769.

4. CABRAL EKF, et al. Efeito Ventilatório da Fisioterapia Intensiva na Síndrome de Guillain-Barré sob Ventilação Mecânica. Revista Brasileira de Ciências da Saúde. 2012; 16 (2): 11-16.

5. CECATTO S, et al. Síndrome de Guillain-Barré como complicação de amigdalite aguda. Revista Brasileira de Otorrinolaringologia, 2003; 69(4): 566-569.

6. DOORN $\mathrm{P}$, et al. Clinical features, pathogenesis, and treatment of Guillain-Barré syndrome. The Lancet Neurology, 2008; 7(10) 939-950.

7. GOLDMAN L, SCHAFER Al. Goldman Cecil Medicina. $24^{a}$ ed. Rio de Janeiro: Elsevier, 2014; 3245p.

8. LONGO DL, et al. Medicina Interna de Harrison. 18 ${ }^{\text {a }}$ ed. Porto Alegre: AMGH, 2013; 2667p.

9. MALTA JMAS, et al. Síndrome de Guillain-Barré e outras manifestações neurológicas possivelmente relacionadas à infecção pelo vírus Zika em municípios da Bahia, 2015. Epidemiologia e Serviços de Saúde. $2017 ; 26(1): 9-18$.

10. NOVIELLO TB, et al. Cetoacidose diabética associada com a síndrome de Guillain-Barré: relato de caso. Arquivos Brasileiros de Endocrinologia \& Metabologia. 2008; 52 (3): 562-565.

11. SANTANA JCB, et al. Distúrbios autonômicos na Síndrome de Guillain-Barré: experiência de 13 anos em UTI pediátrica. Jornal de Pediatria. 1996; 72 (1): 20-26.

12. SEJVAR JJ, et al. Population incidence of Guillain-Barré syndrome: a systematic review and meta-analysis. Neuroepidemiology. 2011; 36(2): 123-33.

13. TORRICELLI RPJE. Fraqueza muscular aguda em crianças. Arquivos de Neuro-Psiquiatria, 2017; 75(4): 248254. 